\title{
Strong exciton-plasmon coupling in semiconducting carbon nanotubes
}

\author{
I.V. Bondarev* \\ Physics Department, North Carolina Central University, \\ 1801 Fayetteville Str, Durham, NC 27707, USA \\ K. Tatur and L.M. Woods \\ Physics Department, University of South Florida, 4202 E.Fowler Ave, Tampa, FL 33620, USA
}

\begin{abstract}
We study theoretically the interactions of excitonic states with surface electromagnetic modes of small-diameter $(\lesssim 1 \mathrm{~nm})$ semiconducting single-walled carbon nanotubes. We show that these interactions can result in strong exciton-surface-plasmon coupling. The exciton absorption lineshapes exhibit the line (Rabi) splitting $\sim 0.1-0.3 \mathrm{eV}$ as the exciton energy is tuned to the nearest interband surface plasmon resonance of the nanotube. We expect this effect to open a path to new optoelectronic device applications of semiconducting carbon nanotubes.

PACS numbers: 78.40.Ri, 73.22.-f, 73.63.Fg, 78.67.Ch
\end{abstract}

Single-walled carbon nanotubes $(\mathrm{CNs})$ are quasi-onedimensional (1D) cylindrical wires consisting of graphene sheets rolled-up into cylinders with diameters $\sim 1-10 \mathrm{~nm}$ and lengths $\sim 1-10^{4} \mu \mathrm{m}[1,2,3]$. CNs are shown to be useful for miniaturized electronic, electromechanical, chemical and scanning probe devices and as materials for macroscopic composites [4]. The area of their potential applications has been recently expanded towards nanophotonics and optoelectronics [5, 6] after the experimental demonstration of controllable single-atom incapsulation into single-walled CNs [7].

For pristine (undoped) single-walled CNs, the numerical calculations predicting large exciton binding energies $(\sim 0.3-0.6 \mathrm{eV})$ in semiconducting $\mathrm{CNs}[8]$ and even in some small-diameter $(\sim 0.5 \mathrm{~nm})$ metallic CNs $[9]$, followed by the results of various measurements of the excitonic photoluminescence [10, 11, 12], have become available. These works, together with other reports investigating the role of effects such as intrinsic defects [1], exciton-phonon interactions 12, 13, 14], external magnetic and electric fields [15, 16], reveal the variety and complexity of the intrinsic optical properties of carbon nanotubes.

Here we develop a theory for the interactions between excitonic states and surface electromagnetic modes in small-diameter $(\lesssim 1 \mathrm{~nm})$ semiconducting single-walled CNs. The approach is based on our recently developed Green function formalism to quantize an electromagnetic field in the presence of quasi-1D absorbing bodies [17, 18, 19]. We show that such interactions can result in a strong exciton-surface-plasmon coupling due to the presence of the low-energy $(\sim 0.5-2 \mathrm{eV})$ weaklydispersive interband plasmon modes [20] and the large exciton excitation energies $\sim 1 \mathrm{eV}[21]$ in small-diameter CNs. This may be used for new CN optoelectronic device applications.

We consider the vacuum-type electromagnetic interaction of an exciton with surface electromagnetic fluctuations of a single-walled semiconducting carbon nanotube.
No external electromagnetic field is assumed to be applied. Since the problem has the cylindrical symmetry, the orthonormal cylindrical basis $\left\{\mathbf{e}_{r}, \mathbf{e}_{\varphi}, \mathbf{e}_{z}\right\}$ is used with the vector $\mathbf{e}_{z}$ directed along the nanotube axis. The total Hamiltonian of the coupled exciton-photon system is of the form (we use Gaussian units)

$$
\hat{H}=\hat{H}_{F}+\hat{H}_{e x}+\hat{H}_{\text {int }},
$$

where the three terms represent the free field, the free exciton, and their interaction, respectively. More explicitly, the second quantized field Hamiltonian is [17, 18, 19]

$$
\hat{H}_{F}=\sum_{\mathbf{n}} \int_{0}^{\infty} d \omega \hbar \omega \hat{f}^{\dagger}(\mathbf{n}, \omega) \hat{f}(\mathbf{n}, \omega)
$$

where the scalar bosonic field operators $\hat{f}^{\dagger}(\mathbf{n}, \omega)$ and $\hat{f}(\mathbf{n}, \omega)$ create and annihilate, respectively, the surface electromagnetic excitation of frequency $\omega$ at an arbitrary point $\mathbf{n}=\mathbf{R}_{n}=\left\{R_{C N}, \varphi_{n}, z_{n}\right\}$ associated with a carbon atom (representing a lattice site) on the surface of the $\mathrm{CN}$ of radius $R_{C N}$. The summation is made over all the carbon atoms, and in the following it is replaced by the integration over the entire nanotube surface according to the rule $\sum_{\mathbf{n}} \ldots=\left(1 / S_{0}\right) \int d \mathbf{R}_{n} \ldots=$ $\left(1 / S_{0}\right) \int_{0}^{2 \pi} d \varphi_{n} R_{C N} \int_{-\infty}^{\infty} d z_{n} \ldots$, where $S_{0}=(3 \sqrt{3} / 4) b^{2}$ is the area of an elementary equilateral triangle selected around each carbon atom in a way to cover the entire surface of the nanotube with $b=1.42 \AA$ being the carboncarbon interatomic distance.

The second quantized Hamiltonian of the free exciton is of the form (see, e.g., Ref. 22])

$$
\hat{H}_{F}=\sum_{\mathbf{n}, f} E_{f}(\mathbf{n}) B_{\mathbf{n}+\mathbf{m}, f}^{\dagger} B_{\mathbf{m}, f}=\sum_{\mathbf{k}, f} E_{f}(\mathbf{k}) B_{\mathbf{k}, f}^{\dagger} B_{\mathbf{k}, f},
$$

where the operators $B_{\mathbf{n}, f}^{\dagger}$ and $B_{\mathbf{n}, f}$ create and annihilate, respectively, an exciton with energy $E_{f}(\mathbf{n})$ at the lattice site $\mathbf{n}$ of the $\mathrm{CN}$ surface. The index $f(\neq 0)$ refers 
to the internal degrees of freedom of the exciton. Alternatively, $B_{\mathbf{k}, f}^{\dagger}=\sum_{\mathbf{n}} B_{\mathbf{n}, f}^{\dagger} e^{i \mathbf{k} \cdot \mathbf{n}} / \sqrt{N}$ creates ( $N$ is the number of the lattice sites) and $B_{\mathbf{k}, f}=\left(B_{\mathbf{k}, f}^{\dagger}\right)^{\dagger}$ annihilates the exciton with quasi-momentum $\mathbf{k}=\left\{k_{\varphi}, k_{z}\right\}$ and energy $E_{f}(\mathbf{k})=\sum_{\mathbf{n}} E_{f}(\mathbf{n}) e^{-i \mathbf{k} \cdot \mathbf{n}} / N=E_{e x c}^{(f)}+\hbar^{2} \mathbf{k}^{2} / 2 M_{e x}$, where $E_{e x c}^{(f)}=E_{b}^{(f)}+E_{g}$ is the $f$-internal-state exciton excitation energy, $E_{b}^{(f)}$ is the corresponding binding energy of the exciton, $M_{e x}=m_{e}+m_{h}$ is its effective translational mass, and $E_{g}$ is the nanotube band gap. The two equivalent free-exciton Hamiltonian representations are related to one another via the obvious orthogonality relationships $\sum_{\mathbf{n}} e^{-i\left(\mathbf{k}-\mathbf{k}^{\prime}\right) \cdot \mathbf{n}} / N=\delta_{\mathbf{k k}^{\prime}}$ and $\sum_{\mathbf{k}} e^{-i(\mathbf{n}-\mathbf{m}) \cdot \mathbf{k}} / N=\delta_{\mathbf{n m}}$ with the $\mathbf{k}$-summation running over the first Brillouin zone of the nanotube. The bosonic field operators in Eq. (2) are transformed to the $\mathbf{k}$-representation in the same way.

The most general (non-relativistic, electric dipole) exciton-photon interaction on the nanotube surface can be written in the form (see Refs. [18, 19])

$$
\begin{gathered}
\hat{H}_{i n t}=\hat{H}_{i n t}^{(1)}+\hat{H}_{i n t}^{(2)} \\
=-\frac{e}{m_{e} c} \sum_{\mathbf{n}} \hat{\mathbf{A}}(\mathbf{n}) \cdot\left[\hat{\mathbf{p}}_{\mathbf{n}}-\frac{e}{2 c} \hat{\mathbf{A}}(\mathbf{n})\right]+\sum_{\mathbf{n}} \hat{\mathbf{d}}_{\mathbf{n}} \cdot \nabla_{\mathbf{n}} \hat{\varphi}(\mathbf{n}),
\end{gathered}
$$

where $\hat{\mathbf{p}}_{\mathbf{n}}=\sum_{f}\left\langle 0\left|\hat{\mathbf{p}}_{\mathbf{n}}\right| f\right\rangle B_{\mathbf{n}, f}+$ h.c. is the total electron momentum operator at the lattice site $\mathbf{n}$ under the optical dipole transition resulting in the exciton formation at the same site, $\hat{\mathbf{d}}_{\mathbf{n}}=\sum_{f}\left\langle 0\left|\hat{\mathbf{d}}_{\mathbf{n}}\right| f\right\rangle B_{\mathbf{n}, f}+$ h.c. is the corresponding dipole moment operator [related to $\hat{\mathbf{p}}_{\mathbf{n}}$ via the equation $\left.\left\langle 0\left|\hat{\mathbf{p}}_{\mathbf{n}}\right| f\right\rangle=i m_{e} E_{f}(\mathbf{n})\left\langle 0\left|\hat{\mathbf{d}}_{\mathbf{n}}\right| f\right\rangle / \hbar e\right]$. The vector potential operator $\hat{\mathbf{A}}(\mathbf{n})$ (the Coulomb gauge is assumed) and the scalar potential operator $\hat{\varphi}(\mathbf{n})$ represent, respectively, the nanotube's transversely polarized surface electromagnetic modes and longitudinally polarized surface electromagnetic modes which the exciton interacts with. We express them in terms of our earlier developed electromagnetic field quantization formalism in the presence of quasi-1D absorbing bodies to obtain [18, 19]

$$
\begin{gathered}
\hat{\mathbf{A}}(\mathbf{n})=\int_{0}^{\infty} d \omega \frac{c}{i \omega} \underline{\hat{\mathbf{E}}}^{\perp}(\mathbf{n}, \omega)+\text { h.c. }=\sum_{\mathbf{m}} \int_{0}^{\infty} d \omega \\
\times \frac{4}{c} \sqrt{\pi \hbar \omega \operatorname{Re} \sigma_{z z}\left(R_{C N}, \omega\right)}{ }^{\perp} G_{z z}(\mathbf{n}, \mathbf{m}, \omega) \hat{f}(\mathbf{m}, \omega)+\text { h.c. }
\end{gathered}
$$

and

$$
\begin{gathered}
-\nabla_{\mathbf{n}} \hat{\varphi}(\mathbf{n})=\int_{0}^{\infty} d \omega \underline{\hat{\mathbf{E}}}^{\|}(\mathbf{n}, \omega)+h . c .=\sum_{\mathbf{m}} \int_{0}^{\infty} d \omega \\
\times \frac{4 i \omega}{c^{2}} \sqrt{\pi \hbar \omega \operatorname{Re} \sigma_{z z}\left(R_{C N}, \omega\right)} \|_{G_{z z}(\mathbf{n}, \mathbf{m}, \omega) \hat{f}(\mathbf{m}, \omega)+\text { h.c. }}
\end{gathered}
$$

with the total electric field operator given by $\hat{\mathbf{E}}(\mathbf{n})=$ $\int_{0}^{\infty} d \omega\left[\underline{\hat{\mathbf{E}}}^{\perp}(\mathbf{n}, \omega)+\underline{\hat{\mathbf{E}}}^{\|}(\mathbf{n}, \omega)\right]+h . c .,{ }^{\perp(\|)} G_{z z}(\mathbf{n}, \mathbf{m}, \omega)$ being the zz-component of the transverse (longitudinal) Green tensor (with respect to the first variable) of the electromagnetic subsystem, and $\sigma_{z z}\left(R_{C N}, \omega\right)$ representing the CN dynamic surface axial conductivity per unit length.

Equations (11)-(6) form the complete set of equations describing the exciton-photon coupled system on the CN surface in terms of the electromagnetic field Green tensor and the $\mathrm{CN}$ surface axial conductivity. The conductivity is found beforehand from the realistic band structure of a particular CN. The Green tensor is derived by expanding the solution of the Green equation in cylindrical coordinates and determining the Wronskian normalization constant from the appropriately chosen boundary conditions on the CN surface (see Refs. [17, 18, 19, 23]). More details on these calculations will be given elsewhere.

It is important to realize that the transversely polarized surface electromagnetic mode contribution to the interaction Hamiltonian from Eq. (4) (first term) is negligible compared to the longitudinally polarized surface electromagnetic mode contribution (second term). The point is that, because of the nanotube quasi-onedimensionality, the exciton quasi-momentum vector and all the relevant vectorial matrix elements of the momentum and dipole moment operators are directed predominantly along the $\mathrm{CN}$ axis (the longitudinal exciton). This prevents the exciton from the electric dipole coupling to transversely polarized surface electromagnetic modes as they propagate predominantly along the $\mathrm{CN}$ axis with their electric vectors orthogonal to the propagation direction. The longitudinally polarized surface electromagnetic modes are generated by the electronic Coulomb potential (see, e.g., Ref. [24]), and therefore represent $\mathrm{CN}$ surface plasmon excitations. These have their electric vectors directed along the propagation direction. They do couple to the longitudinal excitons on the $\mathrm{CN}$ surface. Such modes were observed in Ref. [20]. They occur in CNs both at high energies (well-known $\pi$-plasmon at $\sim 6 \mathrm{eV}$ ) and at comparatively low energies of $\sim 0.5-2 \mathrm{eV}$ [plasmon modes associated with (transversely quantized) interband electronic transitions].

To obtain the dispersion equation of the coupled exciton-plasmon excitations, we utilize Bogoliubov's canonical transformation technique (see, e.g., Ref. 25]) and diagonalize the Hamiltonian (10)-(何) exactly. This results in

$$
\hat{H}=\sum_{\mathbf{k}, \mu=1,2} \hbar \omega_{\mu}(\mathbf{k}) \hat{\xi}_{\mu}^{\dagger}(\mathbf{k}) \hat{\xi}_{\mu}(\mathbf{k})+E_{0}
$$

where $\hat{\xi}_{\mu}(\mathbf{k})=\sum_{f}\left[u_{\mu}^{*}\left(\mathbf{k}, \omega_{f}\right) B_{\mathbf{k}, f}-v_{\mu}\left(\mathbf{k}, \omega_{f}\right) B_{-\mathbf{k}, f}^{\dagger}\right]+$ $\int_{0}^{\infty} d \omega\left[u_{\mu}(\mathbf{k}, \omega) \hat{f}(\mathbf{k}, \omega)-v_{\mu}^{*}(\mathbf{k}, \omega) \hat{f}^{\dagger}(-\mathbf{k}, \omega)\right]$ annihilates and $\hat{\xi}_{\mu}^{\dagger}(\mathbf{k})=\left[\hat{\xi}_{\mu}(\mathbf{k})\right]^{\dagger}$ creates the coupled exciton-plasmon excitations. Here $\mu$ enumerates the two exciton-plasmon branches, $u_{\mu}$ and $v_{\mu}$ are the (appropriately chosen) canonical transformation coefficients, $\omega_{f}=E_{f} / \hbar$. The energy $E_{0}$ is the "vacuum" energy with no excitonplasmons excited in the system, and $\hbar \omega_{\mu}(\mathbf{k})$ is the 
exciton-plasmon energy given by the solution of the following (dimensionless) dispersion equation

$$
x_{\mu}^{2}-\varepsilon_{f}^{2}-\frac{\varepsilon_{f}}{2 \pi} \int_{0}^{\infty} d x x \bar{\Gamma}_{0}^{f}(x) \frac{\rho(x)}{x_{\mu}^{2}-x^{2}}=0 .
$$

Here $x=\hbar \omega / 2 \gamma_{0}, x_{\mu}=\hbar \omega_{\mu}(\mathbf{k}) / 2 \gamma_{0}, \varepsilon_{f}=E_{f}(\mathbf{k}) / 2 \gamma_{0}$ with $\gamma_{0}=2.7 \mathrm{eV}$ being the carbon nearest neighbor overlap integral entering the CN surface axial conductivity $\sigma_{z z}$. The function $\bar{\Gamma}_{0}^{f}(x)=\left(2 \gamma_{0} / \hbar\right)^{2}\left(4\left|d_{f}\right|^{2} x^{3} / 3 \hbar c^{3}\right)$, where $d_{f}=\sum_{\mathbf{n}}\left\langle 0\left|\hat{\mathbf{d}}_{\mathbf{n}}\right| f\right\rangle$, represents the (dimensionless) spontaneous decay rate, and

$$
\rho(x)=\frac{3 S_{0}}{4 \pi \alpha R_{C N}^{2}} \operatorname{Re} \frac{1}{\bar{\sigma}_{z z}(x)}
$$

stands for the surface plasmon density of states (DOS) responsible for the decay rate variation due to the coupling to plasmon modes. Here $\alpha=e^{2} / \hbar c=1 / 137$ is the fine-structure constant and $\bar{\sigma}_{z z}=2 \pi \hbar \sigma_{z z} / e^{2}$ is the dimensionless $\mathrm{CN}$ surface axial conductivity per unit length. The conductivity factor equals $\operatorname{Re}\left(1 / \sigma_{z z}\right)=$ $-\left(8 \pi / \omega R_{C N}\right) \operatorname{Im}\left[1 /\left(\epsilon_{z z}-1\right)\right.$ in view of the Drude relationship $\sigma_{z z}=-i \omega\left(\epsilon_{z z}-1\right) / 4 \pi S \rho_{T}$, where $\epsilon_{z z}$ is the longitudinal (along the CN axis) dielectric function, $S$ and $\rho_{T}$ are the surface area of the tubule and the number of tubules per unit volume, respectively [17, 18, 19]. This relates very closely the surface plasmon DOS function (9) to the loss function $-\operatorname{Im}(1 / \epsilon)$ measured in Electron Energy Loss Spectroscopy (EELS) experiments to determine the properties of collective electronic excitations [20].

The calculated plasmon DOS functions are shown in the left panels of Fig. 1 (a), (b) and (c), along with the functions $\operatorname{Re} \bar{\sigma}_{z z}(x)$ and $\operatorname{Im} \bar{\sigma}_{z z}(x)$, for the $(11,0)$, $(10,0)$ and $(8,0)$ CN we study here $\left(R_{C N}=0.43,0.39\right.$ and $0.31 \mathrm{~nm}$, respectively). In all graphs the lower dimensionless energy limits are set up to equal the lowest bright exciton excitation energy $\left[E_{\text {exc }}=1.21,1.00\right.$ and $1.55 \mathrm{eV}$ for $(11,0),(10,0)$ and $(8,0) \mathrm{CN}$ [21], respectively]. Peaks of the plasmon DOS are seen to coincide in energy with the zeros of the imaginary conductivities (or the zeros of the real dielectric functions), clearly indicating the plasmonic nature of the $\mathrm{CN}$ surface excitations under consideration. Peaks broaden as the $\mathrm{CN}$ diameters decrease. This is consistent with the stronger hybridization effects in smaller-diameter CNs 26$]$. The $\mathrm{CN}$ dielectric response is calculated in the following way. First, we adapt the nearest-neighbor non-orthogonal tight-binding approach [27] to determine the realistic band structure of each CN. Then, the room-temperature longitudinal dielectric function and conductivity are calculated within the random-phase approximation with electronic dissipation processes included in the relaxation-time approximation (electron scattering length of $130 R_{C N}$ was used [14]).

We further take advantage of the peak structure of $\rho(x)$ and solve Eq. (8) analytically using the Lorentzian
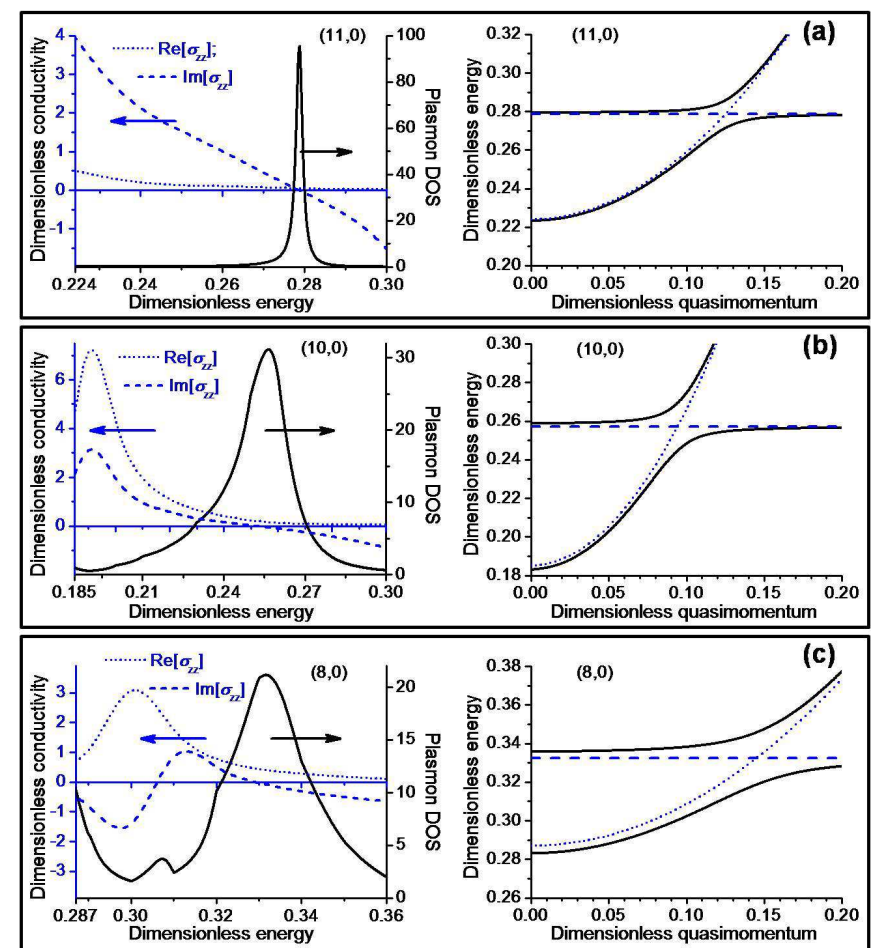

FIG. 1: (Color online) (a),(b),(c) Surface plasmon DOS and conductivities (left panels), and lowest bright exciton dispersion when coupled to plasmons (right panels) in $(11,0),(10,0)$ and $(8,0) \mathrm{CN}$, respectively. Dimensionless energy is defined as [Energy $] / 2 \gamma_{0}$; see text for dimensionless quasimomentum.

approximation with $\rho(x) \approx \rho\left(x_{p}\right) \Delta x_{p}^{2} /\left[\left(x-x_{p}\right)^{2}+\Delta x_{p}^{2}\right]$, where $x_{p}$ and $\Delta x_{p}$ are, respectively, the position and the half-width-at-half-maximum of the plasmon resonance (Fig. 1, left panels) closest to the lowest bright exciton excitation energy in the same nanotube. We obtain

$$
x_{1,2}=\sqrt{\frac{\varepsilon_{f}^{2}+x_{p}^{2}}{2} \pm \frac{1}{2} \sqrt{\left(\varepsilon_{f}^{2}-x_{p}^{2}\right)^{2}+F_{p} \varepsilon_{f}}},
$$

$F_{p}=F\left(x_{p}\right) \Delta x_{p}\left(1-\Delta x_{p} / \pi x_{p}\right), F\left(x_{p}\right)=2 x_{p} \bar{\Gamma}_{0}^{f}\left(x_{p}\right) \rho\left(x_{p}\right)$. The dispersion curves thus obtained are shown in the right panels of Fig. 1 (a), (b) and (c) as functions of the longitudinal dimensionless quasi-momentum. All graphs demonstrate a clear anticrossing behavior of the two exciton-plasmon branches with the (Rabi) energy splitting $\sim 0.1 \mathrm{eV}$. In these calculations, we estimated the interband transition matrix element in $\bar{\Gamma}_{0}^{f}\left(x_{p}\right)$ from the relationship $\left|d_{f}\right|^{2}=3 \hbar \lambda^{3} / 4 \tau_{e x}^{r a d}$ [28], where $\tau_{e x}^{r a d}$ is the exciton intrinsic radiative lifetime, $\lambda=2 \pi c \hbar / E$ with $E=E_{\text {exc }}+(2 \pi \hbar / 3 b)^{2} t^{2} / 2 M_{\text {ex }}$ being the total energy and $-1 \leq t \leq 1$ representing the longitudinal dimensionless quasi-momentum of the exciton. We used the lowest bright exciton parameters reported in Ref. 21].

We also derive the (dimensionless) exciton absorption lineshape function $I(x)$ in the vicinity of the plasmon resonance in the way this was done in Ref. [5] for the 

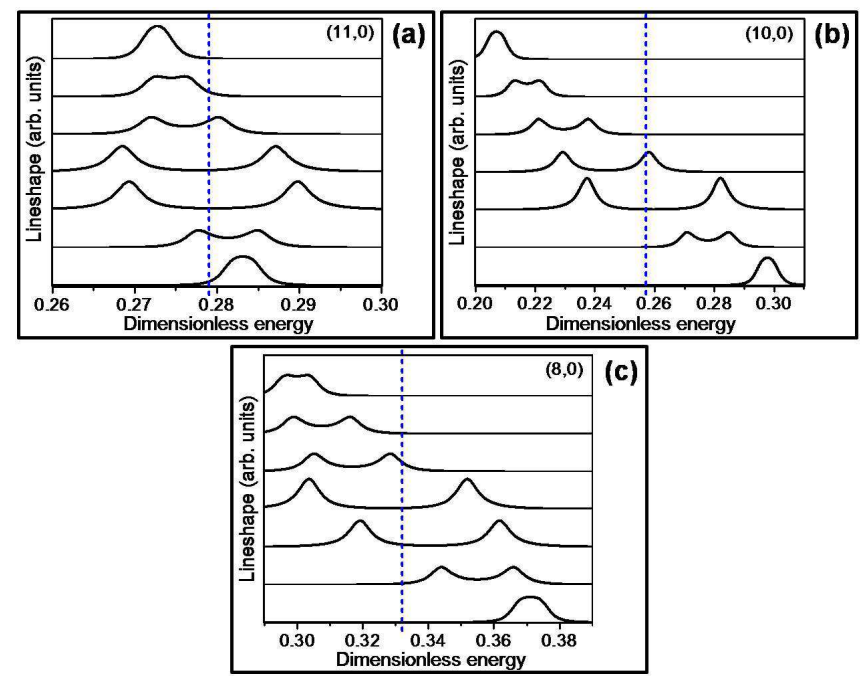

FIG. 2: (Color online) (a),(b),(c) Exciton absorption lineshapes as the exciton energies are tuned to the nearest plasmon resonance energies (vertical dashed lines here; see Fig. 1 left panels) in the $(11,0),(10,0)$ and $(8,0) \mathrm{CN}$, respectively.

optical absorption by atomically doped CNs. In doing so, we take into account the exciton-phonon scattering in the relaxation time approximation. We obtain

$$
I(x)=I_{0}\left(\varepsilon_{f}\right) \frac{\left(x-\varepsilon_{f}\right)^{2}+\Delta_{1}^{2}}{\left[\left(x-\varepsilon_{f}\right)^{2}-X_{f}^{2} / 4\right]^{2}+\left(x-\varepsilon_{f}\right)^{2} \Delta_{2}^{2}},
$$

where $I_{0}\left(\varepsilon_{f}\right)=\bar{\Gamma}_{0}^{f}\left(\varepsilon_{f}\right) \rho\left(\varepsilon_{f}\right) / 2 \pi, X_{f}=\sqrt{4 \pi \Delta x_{p} I_{0}\left(\varepsilon_{f}\right)}$, $\Delta_{1}=\sqrt{2 \Delta \varepsilon_{f}+\Delta x_{p}}$, and $\Delta_{2}=\sqrt{\left(\Delta \varepsilon_{f}\right)^{2}+\Delta_{1}^{2}}$ with $\Delta \varepsilon_{f}=\hbar / 2 \gamma_{0} \tau_{p h}$ being the exciton energy broadening due to the phonon scattering with the relaxation time $\tau_{p h}$. The calculated exciton absorption lineshapes for the CNs under consideration are shown in Fig. 2 (a), (b) and (c) as the exciton energies are tuned to the nearest plasmon resonances. We used $\tau_{p h}=30 \mathrm{fs}$ as reported in Ref. [13]. The clear line (Rabi) splitting effect is seen to be of the order of $0.1-0.3 \mathrm{eV}$, indicating the strong excitonplasmon coupling with the formation of the mixed surface plasmon-exciton excitations. The splitting is larger in the smaller diameter nanotubes, and is not masked by the exciton-phonon scattering.

In summary, we have shown the strong exciton-surfaceplasmon coupling effect with characteristic Rabi splitting $\sim 0.1-0.3 \mathrm{eV}$ in small-diameter $(\lesssim 1 \mathrm{~nm})$ semiconducting CNs. This is almost as large as typical exciton binding energies in such CNs $(\sim 0.3-0.6 \mathrm{eV}[8])$, and of the same order of magnitude as the exciton-plasmon Rabi splitting in organic semiconductors $(\sim 180 \mathrm{meV}[29])$. Also, this is much larger than the exciton-polariton Rabi splitting in semiconductor microcavities $(\sim 140-400 \mu \mathrm{eV}[30])$, or the exciton-plasmon Rabi splitting in hybrid semiconductormetal nanoparticle molecules [31]. However, the formation of the strongly coupled exciton-plasmon states is only possible if the exciton total energy is in resonance with the energy of an interband surface plasmon mode.
That is not always the case experimentally. To realize the strong coupling effect, the exciton energy should be tuned to the nearest plasmon resonance in ways used for the excitons in semiconductor microcavities - thermally 30. (by elevating sample temperature), and/or electrostatically [32] (via the quantum confined Stark effect with an external electrostatic field applied perpendicular to the nanotube axis). This opens paths to new optoelectronic device applications of semiconducting CNs.

The work is supported by NSF (grant ECS-0631347). K.T. and L.M.W. are supported by DOE (grant DEFG02-06ER46297).

* Corresponding author. E-mail: ibondarev@nccu.edu

[1] R.Saito, G.Dresselhaus, and M.S.Dresselhaus, Science of Fullerens and Carbon Nanotubes (Imperial College Press, London, 1998).

[2] H.Dai, Surf. Sci. 500, 218 (2002).

[3] L.X.Zheng et al., Nature Materials 3, 673 (2004).

[4] R.H.Baughman, A.A.Zakhidov, and W.A.de Heer, Science 297, 787 (2002).

[5] I.V.Bondarev, B.Vlahovic, Phys.Rev.B74, 073401(2006).

[6] I.V.Bondarev, B.Vlahovic, Phys.Rev.B75, 033402(2007).

[7] G.-H.Jeong et al., Phys. Rev. B 68, 075410(2003); Thin Solid Films 435, 307 (2003).

[8] T.Pedersen, Phys. Rev. B 67, 073401 (2003); Carbon 42, 1007 (2004).

[9] C.D.Spataru et al., Phys. Rev. Lett. 92, 077402 (2004).

[10] F.Wang et al., Phys. Rev. Lett. 92, 177401 (2004); Science 308, 838 (2005).

[11] A.Hagen et al., Phys. Rev. Lett. 95, 197401 (2005).

[12] F.Plentz et al., Phys. Rev. Lett. 95, 247401 (2005).

[13] V.Perebeinos, J.Tersoff, and Ph.Avouris, Phys. Rev. Lett. 94, 027402 (2005).

[14] M.Lazzeri et al., Phys. Rev. Lett. 95, 236802 (2005).

[15] S.Zaric et al., Phys. Rev. Lett. 96, 016406 (2006).

[16] V.Perebeinos and Ph.Avouris, Nano Lett. 7, 609 (2007).

[17] I.V.Bondarev and Ph.Lambin, Phys. Rev. B 70, 035407 (2004); Phys.Lett. A 328, 235 (2004).

[18] I.V.Bondarev and Ph.Lambin, Phys. Rev. B 72, 035451 (2005); Solid State Commun. 132, 203 (2004).

[19] I.V.Bondarev and Ph.Lambin, in: Trends in Nanotubes Research (Nova Science, New York, 2006).

[20] T.Pichler et al., Phys. Rev. Lett. 80, 4729 (1998).

[21] C.D.Spataru et al., Phys. Rev. Lett. 95, 247402 (2005).

[22] H.Haken, Quantum Field Theory of Solids, (NorthHolland, Amsterdam, 1976).

[23] J.D. Jackson, Classical Electrodynamics (Wiley, New York, 1975).

[24] L.D.Landau, E.M.Lifshits, The Classical Theory of Fields (Pergamon, New York, 1975).

[25] A.S. Davydov, Quantum Mechanics (Pergamon, New York, 1976).

[26] X.Blase et al., Phys. Rev. Lett. 72, 1878 (1994).

[27] V.N.Popov, L.Henrard, Phys. Rev. B 70, 115407 (2004).

[28] E.Hanamura, Phys. Rev. B 38, 1228 (1998).

[29] J.Bellessa et al., Phys. Rev. Lett. 93, 036404 (2004).

[30] J.P.Reithmaier et al., Nature 432, 197 (2004); T.Yoshie et al., Nature 432, 200 (2004). 
[31] W.Zhang, A.O.Govorov, and G.W.Bryant, Phys. Rev. Lett. 97, 146804 (2006).

[32] A.Zrenner et al., Nature 418, 612 (2002). 